\title{
Quantitative CT-based structural alterations of segmental airways in cement dust- exposed subjects
}

Taewoo Kim${ }^{1}$, Hyun Bin Cho ${ }^{1}$, Woo Jin Kim², Chang Hyun Lee ${ }^{3,4}$, Kum Ju Chae ${ }^{5}$, So-Hyun Choi ${ }^{6}$, Kyeong Eun Lee ${ }^{6}$, So Hyeon Bak', Sung Ok Kwon², Gong Yong Jin ${ }^{5}$, Jiwoong Choi ${ }^{7}$, Eun-Kee Park ${ }^{8}$, Ching-Long Lin ${ }^{7}$, Eric A. Hoffman ${ }^{4}$ and Sanghun Choi ${ }^{1^{*}}$ (D)

\begin{abstract}
Background: Dust exposure has been reported as a risk factor of pulmonary disease, leading to alterations of segmental airways and parenchymal lungs. This study aims to investigate alterations of quantitative computed tomography (QCT)-based airway structural and functional metrics due to cement-dust exposure.

Methods: To reduce confounding factors, subjects with normal spirometry without fibrosis, asthma and pneumonia histories were only selected, and a propensity score matching was applied to match age, sex, height, smoking status, and pack-years. Thus, from a larger data set $(N=609)$, only 41 cement dust-exposed subjects were compared with 164 non-cement dust-exposed subjects. QCT imaging metrics of airway hydraulic diameter $\left(D_{h}\right)$, wall thickness $(\mathrm{WT})$, and bifurcation angle $(\theta)$ were extracted at total lung capacity (TLC) and functional residual capacity (FRC), along with their deformation ratios between TLC and FRC.

Results: In TLC scan, dust-exposed subjects showed a decrease of $D_{h}$ (airway narrowing) especially at lower-lobes $(p<0.05)$, an increase of WT (wall thickening) at all segmental airways $(p<0.05)$, and an alteration of $\theta$ at most of the central airways $(p<0.001)$ compared with non-dust-exposed subjects. Furthermore, dust-exposed subjects had smaller deformation ratios of WT at the segmental airways $(p<0.05)$ and $\theta$ at the right main bronchi and left main bronchi $(p<0.01)$, indicating airway stiffness.
\end{abstract}

Conclusions: Dust-exposed subjects with normal spirometry demonstrated airway narrowing at lower-lobes, wall thickening at all segmental airways, a different bifurcation angle at central airways, and a loss of airway wall elasticity at lower-lobes. The airway structural alterations may indicate different airway pathophysiology due to cement dusts.

Keywords: Airway narrowing, Wall thickening, Bifurcation angle, Stiffness of airway structure, Percent emphysema

\footnotetext{
* Correspondence: s-choi@knu.ac.kr

'School of Mechanical Engineering, Kyungpook National University, 80

Daehak-ro, Buk-gu, Daegu 41566, South Korea

Full list of author information is available at the end of the article
}

(C) The Author(s). 2020 Open Access This article is licensed under a Creative Commons Attribution 4.0 International License, which permits use, sharing, adaptation, distribution and reproduction in any medium or format, as long as you give appropriate credit to the original author(s) and the source, provide a link to the Creative Commons licence, and indicate if changes were made. The images or other third party material in this article are included in the article's Creative Commons licence, unless indicated otherwise in a credit line to the material. If material is not included in the article's Creative Commons licence and your intended use is not permitted by statutory regulation or exceeds the permitted use, you will need to obtain permission directly from the copyright holder. To view a copy of this licence, visit http://creativecommons.org/licenses/by/4.0/ The Creative Commons Public Domain Dedication waiver (http://creativecommons.org/publicdomain/zero/1.0/) applies to the data made available in this article, unless otherwise stated in a credit line to the data. 


\section{Background}

Dust exposure has been reported as a risk factor for pulmonary disease. For example, occupational dust exposure has been significantly associated with chronic obstructive pulmonary disease (COPD) [1]. Exposure to desert dust has been correlated with an increased risk of hospitalization for asthma [2]. An association between dust exposure and lung function has been reported via cytological and spirometry findings. In dusty areas near cement plants, the serum mercury level of blood samples was correlated with a decrease in the forced expiratory volume in $1 \mathrm{~s}\left(\mathrm{FEV}_{1}\right)$ and a risk of obstructive lung disease [3]. In addition, workers exposed to dust working in a cement factory were likely to have a decrease in peak expiratory flow [4]. However, the effects of environmental dust exposure on residents near cement plants have not been studied in detail. In this study, we hypothesize that environmental dust exposure by cements is associated with alterations of quantitative computed tomography (QCT)based airway structural and functional metrics. Thus, QCT imaging-based variables are used to investigate structural and functional alterations due to dust exposure.

With respect to QCT imaging, few studies have investigated the effects of dust exposure on airway structure and lung function. For instance, coal and gold miners have been found to have a higher prevalence of emphysema compared with control groups [5]. The emphysema score measured by QCT has been associated with construction workers who are heavily exposed to asbestos [6] but not quartz and silica [7, 8]. Many previous studies have been limited regarding fully understanding the effects of dust exposure because they employed only one or a few imaging variables for a small number of subjects. More recently, Marchetti et al. [9] demonstrated that occupational dust-exposed subjects had a greater percentage of emphysema, percentage of air trapping, and wall area. The advanced post-processing of QCT imaging can reveal more airway structure features, such as airway luminal hydraulic diameter $\left(D_{\mathrm{h}}\right)$, wall thickness (WT), and bifurcation angle $(\theta)$ in proximal airways, as well as parenchymal functional features, including air volume, tissue volume, the determinant of Jacobian (Jacobian), percent functional small airway disease (fSAD\%), and percent emphysema (Emph\%), through the image registration technique [10]. QCT metrics were able to classify clinically meaningful clusters of asthma [11].

With a comprehensive set of QCT imaging-based metrics, we aim to investigate unique features of airway structure and lung parenchymal function between subjects exposed to cement dust (dust-exposed: DE) and subjects with none or little exposure to cement dust (non-dust-exposed: NDE). The DE and NDE subjects were acquired at two different imaging sites, respectively. Both imaging sites collected two CT images for a subject at functional residual capacity (FRC) and total lung capacity (TLC). To minimize the intersite variability, we employed a fractionthreshold method $[10,12]$, when estimating parametric response map, i.e., fSAD\% and Emph\%. Next, to control the intersubject variability due to sex, age, height, smoking history, pack-years, and more, we employed a statistical method, i.e., propensity score matching method [13]. This allows for an objective comparison between two groups.

\section{Methods \\ Study population}

We employed 311 subjects with cement dust effects collected from Kangwon National University Hospital (KNUH) supported by a Korean research project called the Chronic Obstructive pulmonary disease in Dusty Areas near cement plants (CODA) cohort over approximately 10 years $[3,14,15]$. This project was designed to investigate the effect of cement-dust exposure on patients' health near cement plants located in the Kangwon and Chungbuk provinces of South Korea considering the distance of cement plants and wind direction based on meteorological data from the National Institute of Environmental Research of the Ministry. The size of cement dust varies between 0.5 and $5 \mu \mathrm{m}$ [16]. As control data, we employed 298 subjects with none or little exposure to cement dust collected from Chonbuk National University Hospital (CNUH) over 3 years [17]. The control subjects had normal findings on CT imaging, such as an absence of lung lesions or air-trapping, and no known history of lung disease or surgery. Both the KNUH and CNUH studies were approved by the Institutional Review Board at individual sites (KNUH 2019-06-007 and CUH 2016-03-020-005) and used a similar imaging protocol (Table 1).

A flow chart for the subject selection procedure is provided in Fig. 1. To select subjects with normal lung function, we only included subjects with $\mathrm{FEV}_{1} /$ forced vital capacity (FVC) $\geq 70 \%$ and FVC \%predicted value $\geq 80 \%$. In addition, we excluded subjects with any prior diagnosis of fibrosis, asthma and/or pneumonia. This exclusion procedure allows for an objective comparison by eliminating confounding effects caused by pulmonary diseases such as fibrosis, asthma, pneumonia, and COPD. Then propensity score matching (PSM) method was applied for $63 \mathrm{DE}$ and 274 NDE subjects, to reduce the confounding effects of age, sex, height, smoking history, and pack-years. See the subsection Statistical analysis for the PSM method. In this study, pulmonary function tests (PFTs) of DE and NDE subjects were performed according to the American Thoracic Society/European Respiratory Society guideline [18]. 
Table 1 Scanners and scanning protocol used for non-dust-exposed subjects and dust-exposed subjects

\begin{tabular}{lll}
\hline & Non-dust-exposed subjects & Dust-exposed subjects \\
\hline Institution & CNUH & KNUH \\
Scanner make & Siemens Definition Flash 128 slices & Siemens Definition AS 64 slices \\
Scan type & Spiral & Spiral \\
Rotation time(s) & 0.5 & 0.5 \\
Detector configuration & $128 \times 0.6 \mathrm{~mm}$ & $64 \times 0.6 \mathrm{~mm}$ \\
Pitch & 1 & 1 \\
Peak kilovoltage, kVp & 120 & 140 \\
mAs & 110, Effective & 100 , Effective \\
Dose modulation & Care dose OFF & Care dose OFF \\
Reconstruction algorithm & B35f & B30f \\
Thickness (mm) & 1 & 0.6 \\
Iterative reconstruction & No selection & No selection \\
\hline
\end{tabular}

CNUH Chonbuk National University Hospital, KNUH Kangwon National University Hospital, mAs Milliamperage seconds

\section{QCT-based airway structure and lung function}

In both TLC and FRC scans, we derived the luminal hydraulic diameter $\left(D_{\mathrm{h}, \text { TLC }}\right.$ and $\left.D_{\mathrm{h}, \text { FRC }}\right)$, airway wall thickness $\left(\mathrm{WT}_{\mathrm{TLC}}\right.$ and $\left.\mathrm{WT}_{\mathrm{FRC}}\right)$, and bifurcation angle $\left(\theta_{\mathrm{TLC}}\right.$ and $\left.\theta_{\mathrm{FRC}}\right)$ using Apollo software 2.0 (VIDA Diagnostics, Coralville, Iowa, USA), along with an in-house post-process. The bifurcation angle was defined as an angle between two daughter branches of a proximal airway. $D_{\mathrm{h}}, \mathrm{WT}$, and $\theta$ could be used to assess airway narrowing, wall thickening, and the alteration of branching structure, respectively.

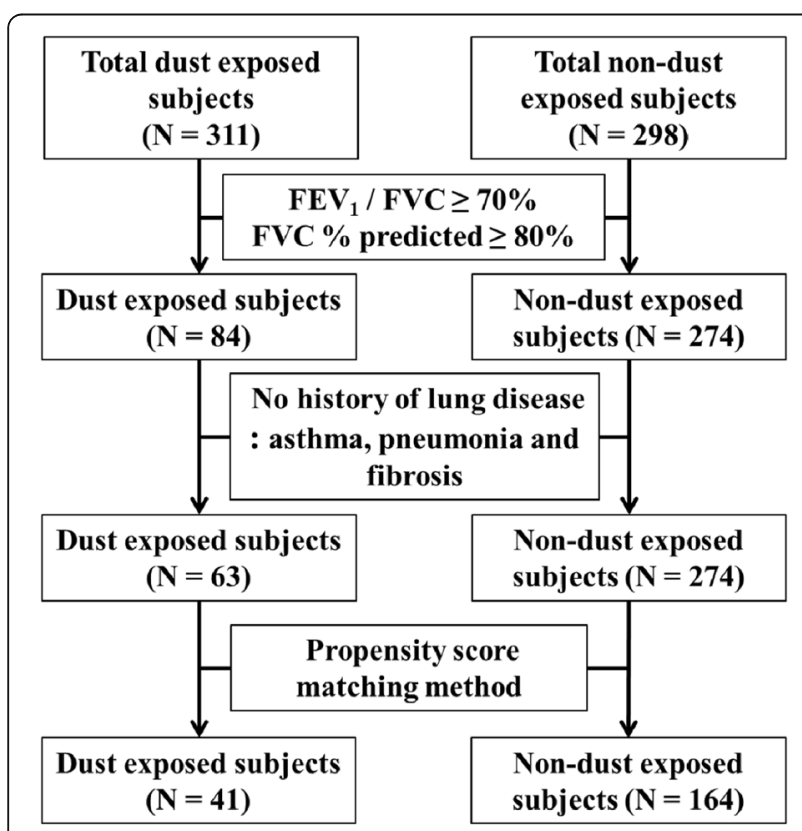

Fig. 1 Flow chart of subject selection for dust-exposed subjects and non-dust-exposed subjects. FEV 1 , forced expiratory volume in $1 \mathrm{~s}$; FVC, forced vital capacity
To assess the deformable features of segmental airways, the deformation ratio $\varepsilon$ between TLC to FRC was computed as follows:

$$
\varepsilon^{\phi}(\%)=\frac{\phi^{T L C}-\phi^{F R C}}{\phi^{F R C}} \times 100,
$$

where $\phi$ is any structural variable of $D_{\mathrm{h}}, \mathrm{WT}$, and $\theta$, so $\varepsilon^{D h}, \varepsilon^{\mathrm{WT}}$, and $\varepsilon^{\theta}$ were derived in this study. To measure the regional features of airways, structural variables were extracted from seven central airways and five subgroup regions. A detailed description of airway labeling is given in Fig. 2. The seven central airways included the trachea, right main bronchus (RMB), bronchus intermedius (BronInt), trifurcation of the right lower lobe (TriRUL), main bronchus (LMB), trifurcation of the left upper lobe (TriLUL), and LLB6. The five subgroup lobes included the right upper lobe (sRUL), right middle lobe (sRML), right lower lobe (sRLL), left upper lobe (sLUL), and left lower lobe (sLLL).

Functional variables included the air volumes at TLC and FRC, inspiratory capacity (IC), and Jacobian between TLC and FRC, respectively. In addition, Emph\% and fSAD\% were computed using an image registration technique [19]. To minimize the inter-center variability of Emph\% and fSAD\%, a fraction-threshold [10] method was used. Detailed formulations of these imaging variables are included in the references $[10,11,20]$.

\section{Statistical analysis}

We used the PSM method to reduce the bias between two groups of DE and NDE subjects. A propensity score is the predicted probability of belonging to the treatment group, and would be calculated for each subject in the study [13]. The propensity scores were estimated by multiple logistic regression analysis using the age and 


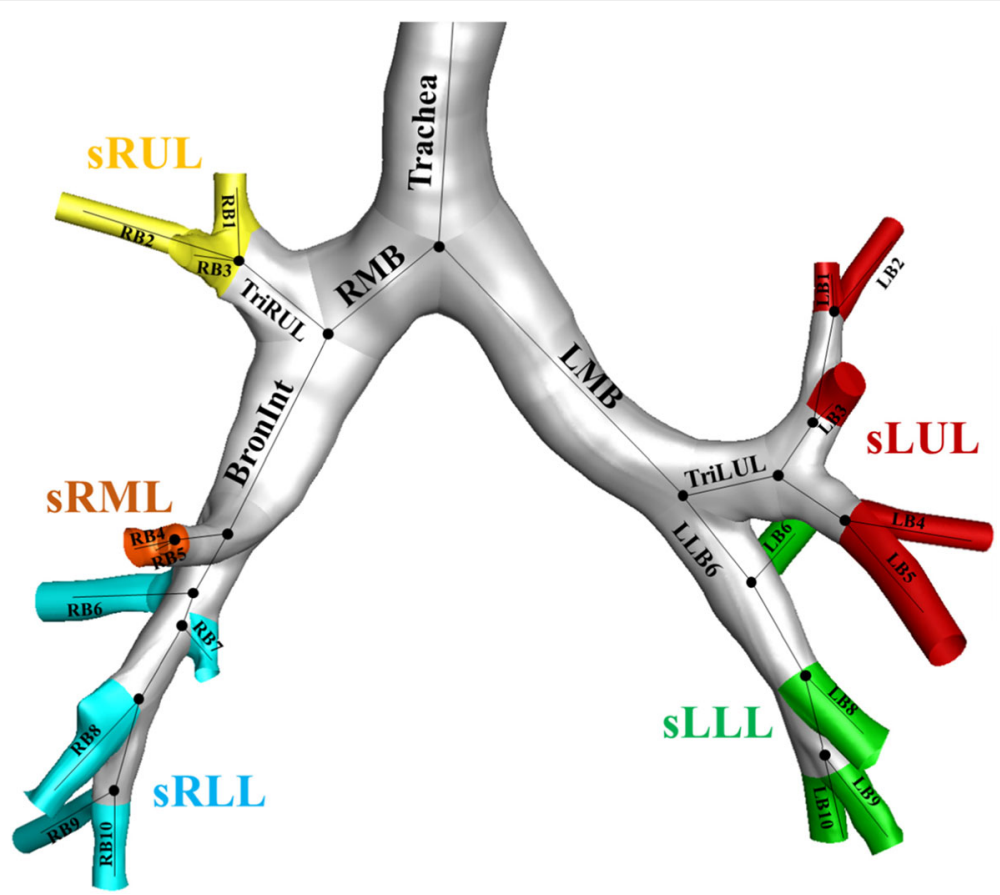

Fig. 2 Labels of 26 segmental airways and 5 subgroups of lobes. Bronlnt, bronchus intermedius; LMB, left main bronchus; RMB, right main bronchus; sLLL, subgrouped left lower lobe including branches of LB6 and LB8 to LB10; sLUL, subgrouped left upper lobe including branches of LB1 to LB5; sRLL, subgrouped right lower lobe including branches of RB6 to RB10; sRML, subgrouped right middle lobe including branches of RB4 and RB5; SRUL, subgrouped right upper lobe including branches of RB1 to RB3; TriLUL, trifurcation of left upper lobe; TriRUL, trifurcation of right upper lobe

Table 2 Propensity score matching before and after data using demographic (sex, age, height, smoking status, and pack-years) information for non-dust-exposed subjects and dust-exposed subjects

\begin{tabular}{|c|c|c|c|c|c|c|c|c|c|c|}
\hline & \multicolumn{5}{|c|}{ Before propensity score matching } & \multicolumn{5}{|c|}{ After propensity score matching (1:4) } \\
\hline & \multirow{2}{*}{\multicolumn{2}{|c|}{$\begin{array}{l}\text { NDE subjects } \\
(N=255)\end{array}$}} & \multirow{2}{*}{\multicolumn{2}{|c|}{$\begin{array}{l}\text { DE subjects } \\
(N=63)\end{array}$}} & \multirow[t]{3}{*}{ St.Diff. } & \multirow{2}{*}{\multicolumn{2}{|c|}{$\begin{array}{l}\text { NDE subjects } \\
(N=164)\end{array}$}} & \multirow{2}{*}{\multicolumn{2}{|c|}{$\frac{\text { DE subjects }}{(N=41)}$}} & \multirow[t]{3}{*}{ St.Diff } \\
\hline & & & & & & & & & & \\
\hline & $\mathrm{N}$ & $(\%)$ & $\mathrm{N}$ & (\%) & & $\mathrm{N}$ & $(\%)$ & $\mathrm{N}$ & (\%) & \\
\hline \multicolumn{11}{|l|}{ Sex } \\
\hline Male & 99 & $(38.8)$ & 45 & $(68.2)$ & 61.6 & 80 & $(48.8)$ & 20 & $(48.8)$ & 0.0 \\
\hline Female & 156 & $(61.2)$ & 21 & (31.8) & & 84 & $(51.2)$ & 21 & $(51.2)$ & \\
\hline \multicolumn{11}{|l|}{ Age } \\
\hline mean \pm std & 51.0 & \pm 15.1 & 70.5 & \pm 7.5 & \pm 1.6 & 49.7 & \pm 15.4 & 70.0 & \pm 8.0 & \pm 1.7 \\
\hline \multicolumn{11}{|l|}{ Height } \\
\hline mean \pm std & 161.4 & \pm 9.6 & 159.9 & \pm 10.1 & \pm 0.2 & 162.9 & \pm 9.7 & 157.9 & \pm 11.3 & \pm 0.5 \\
\hline \multicolumn{11}{|l|}{ Smoking } \\
\hline Non/Former Smoking Participants & 224 & $(87.8)$ & 57 & (86.4) & 4.4 & 144 & $(87.8)$ & 36 & $(87.8)$ & 0.0 \\
\hline Current Smoking Participants & 31 & $(12.2)$ & 9 & (13.6) & & 20 & $(12.2)$ & 5 & $(12.2)$ & \\
\hline \multicolumn{11}{|l|}{ Pack-years } \\
\hline mean \pm std & 4.6 & \pm 13.2 & 14.7 & \pm 21.9 & \pm 0.6 & 4.4 & \pm 12.3 & 10.4 & \pm 22.7 & \pm 0.3 \\
\hline \multicolumn{11}{|l|}{ BMI } \\
\hline mean \pm std & - & & - & & - & 24.4 & \pm 3.3 & 24.1 & \pm 2.8 & $0.372^{*}$ \\
\hline
\end{tabular}

19 subjects were excluded by missing values of pack-years in NDE subjects. NDE Non-dust exposed, $D E$ Dust exposed, St. Diff. Standardized differences; ${ }^{*}, P$-value obtained by one-sample t-test 
Table 3 Comparison of deformation ratios of the bifurcation angle $\left(\varepsilon^{\theta}\right)$ between non-dust-exposed subjects and dustexposed subjects

\begin{tabular}{llllll}
\hline Region & \multicolumn{2}{l}{ NDE subjects $(n=164)$} & \multicolumn{2}{l}{ DE subjects $(n=41)$} & $P$ value \\
\hline Trachea & -2.713 & $(7.232)$ & -1.713 & $(5.389)$ & 0.191 \\
RMB & 14.51 & $(10.44)$ & 8.172 & $(6.396)$ & $<0.001$ \\
TriRUL & 1.290 & $(12.31)$ & 4.194 & $(7.858)$ & $<0.05$ \\
Bronlnt & -2.171 & $(13.53)$ & -1.849 & $(11.07)$ & 0.815 \\
LMB & 11.29 & $(10.40)$ & 7.728 & $(12.39)$ & $<0.01$ \\
TriLUL & 6.366 & $(9.857)$ & 2.708 & $(9.361)$ & $<0.005$ \\
LLB6 & 6.505 & $(13.91)$ & 5.084 & $(10.14)$ & 0.266 \\
\hline
\end{tabular}

Values are presented as mean (SD); Bron/nt Bronchus intermedius, LMB Left main bronchus, RMB Right main bronchus, TriRUL Trifurcation of right lower lobe, TriLUL Trifurcation of left upper lobe, $\varepsilon^{\theta}$ Deformation ratio of

bifurcation angle

height variables stratified sex and smoking status. Matching was done using the Greedy 1-4 matching within a caliper, 0.2 times standard error of propensity scores. To validate PSM we used standardized difference, defined balance as an absolute value less than 10 [21]. PSM method was conducted using SAS 9.4 software.

One sample t-tests were performed for the subjects matched by PSM method to compare the difference of QCT imaging-based metrics. The values are represented by means and the standard deviation (SD) in Tables 2, 3 and 4 and means and the confidence interval (CI) in Figs. 3, 4, 5 and 6. A significance level of $p=0.05$ was chosen for a total of 135 comparison tests, resulting in a false discovery rate of $8.2 \%$. Statistical analyses were conducted using $R$ software.

\section{Results}

\section{Demographics information}

Table 2 shows the demographic information for DE and NDE subjects in the before- and after- propensity score based matched data. The standardized differences of sex and smoking status were zero, indicating an exact matching. Standardized differences of continuous metrics, i.e., age, height, and pack-years were $1.4,0.5$, and 0.3 , respectively. Note that the value of standardized difference smaller than 10 is considered as well-balanced. After matching the continuous metrics, body mass index (BMI) of two groups is also shown to be balanced.

\section{Segmental airways of dust-exposed subjects at TLC and \\ FRC}

Figure 3 shows the generational (left column) and regional (middle and right columns) differences in $D_{\mathrm{h}}$ between DE and NDE subjects from TLC scans $\left(D_{\mathrm{h}}\right.$, TLC; top row) and FRC scans ( $D_{\mathrm{h}}$, FRC; bottom row). Relative to NDE subjects, the $D_{\mathrm{h}}$, TLC and $D_{\mathrm{h}}$, FRC of DE subjects were smaller in the airways at RMB, TriLUL, and TriRUL (Fig. 3b and e). The significant difference of $D_{\mathrm{h}}$ was observed especially at all lobes in TLC $(P<0.05)$. Except for these airways, there were no or little statistical difference of the $D_{\mathrm{h}}$, TLC and $D_{\mathrm{h}}$, FRC.

Figure 4 then shows the generational and regional differences of WT from TLC (top row) and FRC (bottom row) scans. DE subjects had increased WT in all regions except for RMB and LMB at the TLC scan and all regions except for LLB6 at the FRC scan. Consequently, WT was significantly increased from 2 nd to 5 th generation $(P<$ 0.001 ), being different from $D_{\mathrm{h}}$. Compared with NDE subjects, the $\theta_{\mathrm{TLC}}$, and $\theta_{\mathrm{FRC}}$ of the trachea and TriRUL were increased, and the $\theta_{\mathrm{TLC}}$ of RMB, LMB, TriLUL and LLB6 was decreased in DE subjects (Fig. 5a, b). Similarly, the $\theta_{\text {FRC }}$ of DE subjects were decreased in RMB, TriLUL, and LLB6 compared with that of NDE subjects (Fig. 5b).

Regarding the deformation ratio of $D_{\mathrm{h}}\left(\varepsilon^{D h}\right)$, there was no statistical difference between the two groups (not reported here). On the other hand, the $\varepsilon^{\mathrm{WT}}$ of the 4th-5th generations and all subgrouped lobes except sRUL and

Table 4 QCT-based lung functions between non-dust-exposed subjects and dust-exposed subjects

\begin{tabular}{|c|c|c|c|c|c|c|}
\hline \multicolumn{2}{|c|}{ QCT-based lung functions } & \multicolumn{2}{|c|}{ NDE subjects $(n=164)$} & \multicolumn{2}{|c|}{ DE subjects $(n=41)$} & \multirow{2}{*}{$\frac{P \text { value }}{<0.001}$} \\
\hline TLC, liter & & 3.902 & $(1.030)$ & 3.477 & $(0.970)$ & \\
\hline FRC, liter & & 2.163 & $(0.705)$ & 1.977 & $(0.741)$ & $<0.05$ \\
\hline IC, liter & & 1.794 & $(0.842)$ & 1.543 & $(0.723)$ & $<0.005$ \\
\hline \multirow[t]{6}{*}{ Jacobian } & Total & 1.690 & $(0.364)$ & 1.617 & $(0.348)$ & 0.058 \\
\hline & LUL & 1.601 & $(0.346)$ & 1.558 & $(0.355)$ & 0.258 \\
\hline & LLL & 1.884 & $(0.452)$ & 1.900 & $(0.469)$ & 0.734 \\
\hline & RUL & 1.581 & $(0.338)$ & 1.440 & $(0.268)$ & $<0.001$ \\
\hline & $\mathrm{RML}$ & 1.465 & $(0.256)$ & 1.391 & $(0.224)$ & $<0.005$ \\
\hline & RLL & 1.861 & $(0.436)$ & 1.834 & $(0.414)$ & 0.562 \\
\hline Emph\% & & 0.017 & $(0.023)$ & 0.010 & $(0.013)$ & $<0.005$ \\
\hline fSAD\% & & 0.087 & $(0.116)$ & 0.080 & $(0.106)$ & 0.554 \\
\hline
\end{tabular}

Values are presented as mean (SD); Emph\% Percent emphysema; FRC Functional residual volume, fSAD\% Percent functional small airway disease, IC Inspiratory capacity, TLC Total lung capacity, QCT Quantitative computed tomography, LLL Left lower lobe, LUL Left upper lobe, RLL Right lower lobe, RML Right middle lobe, RUL Right upper lobe; The values are presented with absolute values in TLC, FRC, and IC, not predicted values 

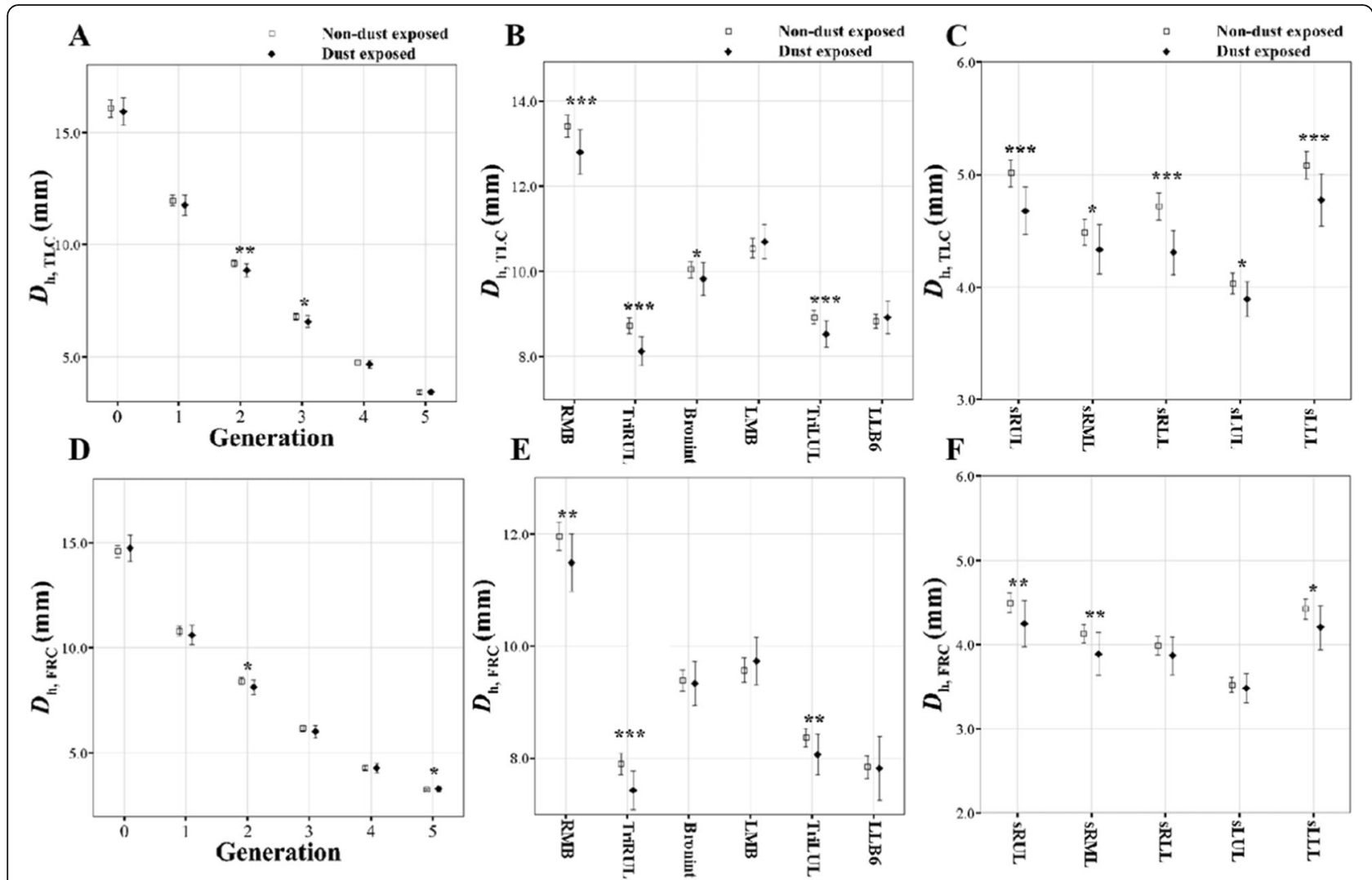

Fig. 3 Comparison of luminal hydraulic diameter $\left(D_{h}\right)$ at TLC $(\mathbf{a}, \mathbf{b}$, and $\mathbf{c})$ and FRC scans $(\mathbf{d}$, e, and $\mathbf{f})$ between non-dust-exposed subjects and dust-exposed subjects. Values are presented as mean $(\mathrm{Cl})$; ${ }^{*} P<0.05$; ${ }^{* *} P<0.01$; ${ }^{* *} P<0.001$. Generation zero is started from trachea. Bronlnt, bronchus intermedius; $D_{h}$, hydraulic diameter; FRC, functional residual capacity; LMB, left main bronchus; RMB, right main bronchus; sLLL, subgrouped left lower lobe; sLUL, subgrouped left upper lobe; sRLL, subgrouped right lower lobe; SRML, subgrouped right middle lobe; sRUL, subgrouped right upper lobe; TLC, Total lung capacity; TriLUL, trifurcation of left upper lobe; TriRUL, trifurcation of right upper lobe

sLLL, namely segmental airways, were significantly smaller in DE subjects than NDE subjects (Fig. 6). Next, DE subjects had significantly smaller $\varepsilon^{\theta}$ in the RMB, LMB, and TriLUL (Table 3). Based upon WT and $\theta$, DE subjects were found to have smaller deformations in bronchial structures when they breathe between TLC and FRC. The quantities possibly indicate an increase in airway stiffness of DE subjects. For instance, Fig. 7 supports this trend of bifurcation angle change at RMB between TLC and FRC in a DE subject (male; 74 years; $\mathrm{BMI}=28$ ) and an $\mathrm{NDE}$ subject (male; 67 years; $\mathrm{BMI}=25$ ).

\section{Functional features of dust-exposed subjects}

Table 4 shows the differences of QCT-based functional metrics between NDE and DE subjects. The values of TLC, FRC, and IC were presented with absolute values, rather than \% predicted values, because sex, age, and height of the data were already balanced by PSM method. Regarding air volumes, both TLC and FRC of DE subjects were smaller, and IC was also smaller in DE subjects than those of NDE subjects. The Jacobian indicating volume change ratio was decreased in $\mathrm{DE}$ subjects at right upper lobe and right middle lobe, consistent with IC. Emph\% of DE subjects was lower than that of NDE subjects. However, $\mathrm{fSAD} \%$ of DE subjects was not significantly different from that of NDE subjects. A figure was displayed to demonstrate parenchymal features of Emph\% and fSAD\% (Fig. 8).

\section{Discussion}

In this study, with the aid of advanced QCT imaging analysis, we have investigated alterations of the airway structure and lung function at multiscale levels in subjects exposed to cement dust. Most similar studies $[1-5,8,9]$ included patients with pulmonary diseases such as COPD and asthma, whereas for an objective comparison, this study excluded patients with pneumonia, asthma, and COPD to minimize confounding effects due to the pulmonary diseases. We also employed a robust statistical method of propensity score matching to control demographic confounders such as age, sex, height, smoking history, and pack-years. It has been known that imaging protocols between different centers are sensitive when estimating density-based imaging metrics such as Emph\%, 

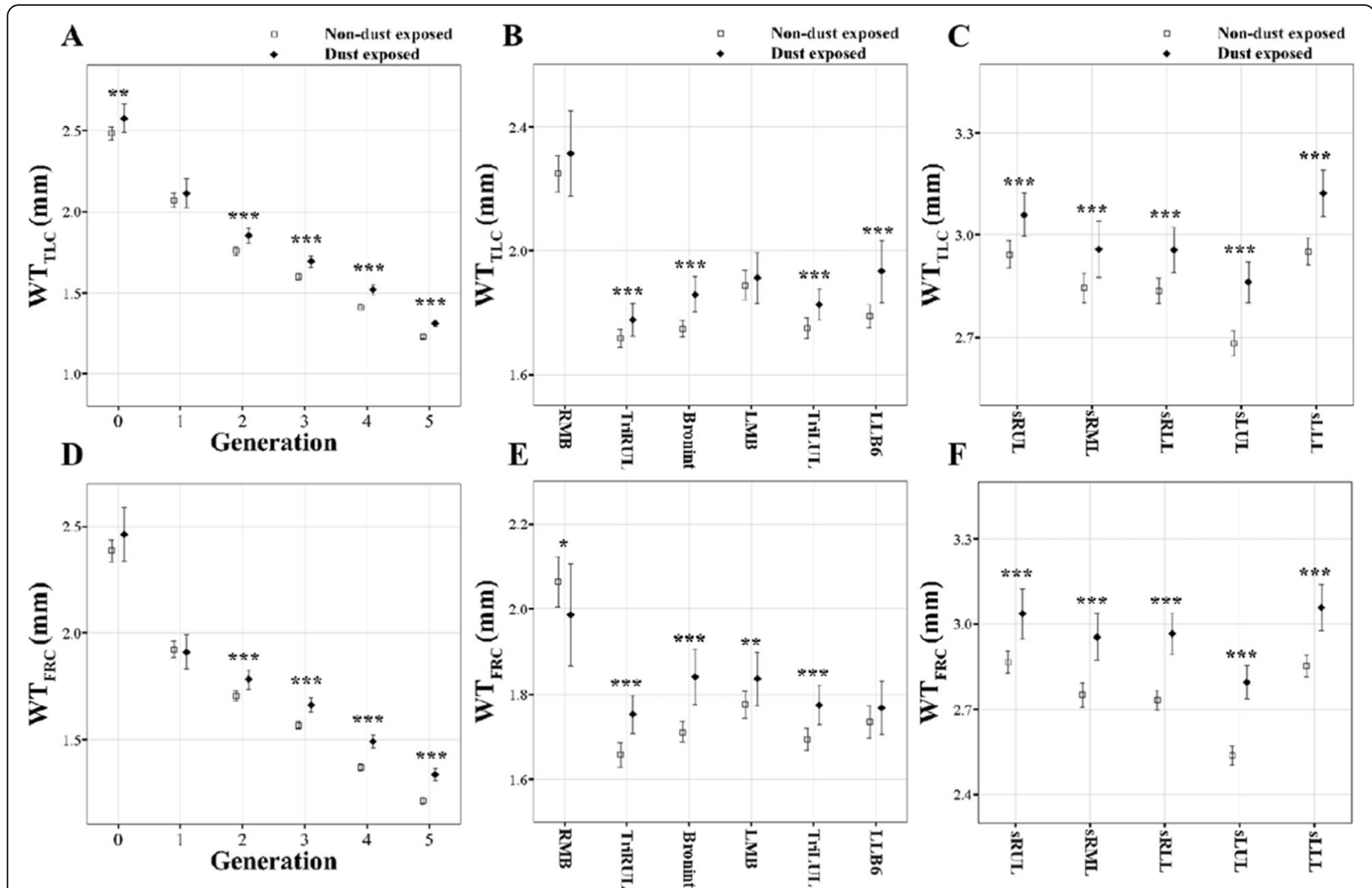

Fig. 4 Comparison of wall thickness (WT) at TLC ( $\mathbf{a}, \mathbf{b}$, and $\mathbf{c}$ ) and FRC scans ( $\mathbf{d}, \mathbf{e}$, and $\mathbf{f}$ ) between non-dust-exposed subjects and dust-exposed subjects. Values are presented as mean $(\mathrm{Cl}) ;{ }^{*} P<0.05 ;{ }^{* *} P<0.01 ;{ }^{* *} P<0.001$. Generation zero is started from trachea. Bronlnt, bronchus intermedius; FRC, functional residual capacity; LMB, left main bronchus; RMB, right main bronchus; sLLL, subgrouped left lower lobe; SLUL, subgrouped left upper lobe; sRLL, subgrouped right lower lobe; SRML, subgrouped right middle lobe; sRUL, subgrouped right upper lobe; TLC, total lung capacity; TriLUL, trifurcation of left upper lobe; TriRUL, trifurcation of right upper lobe

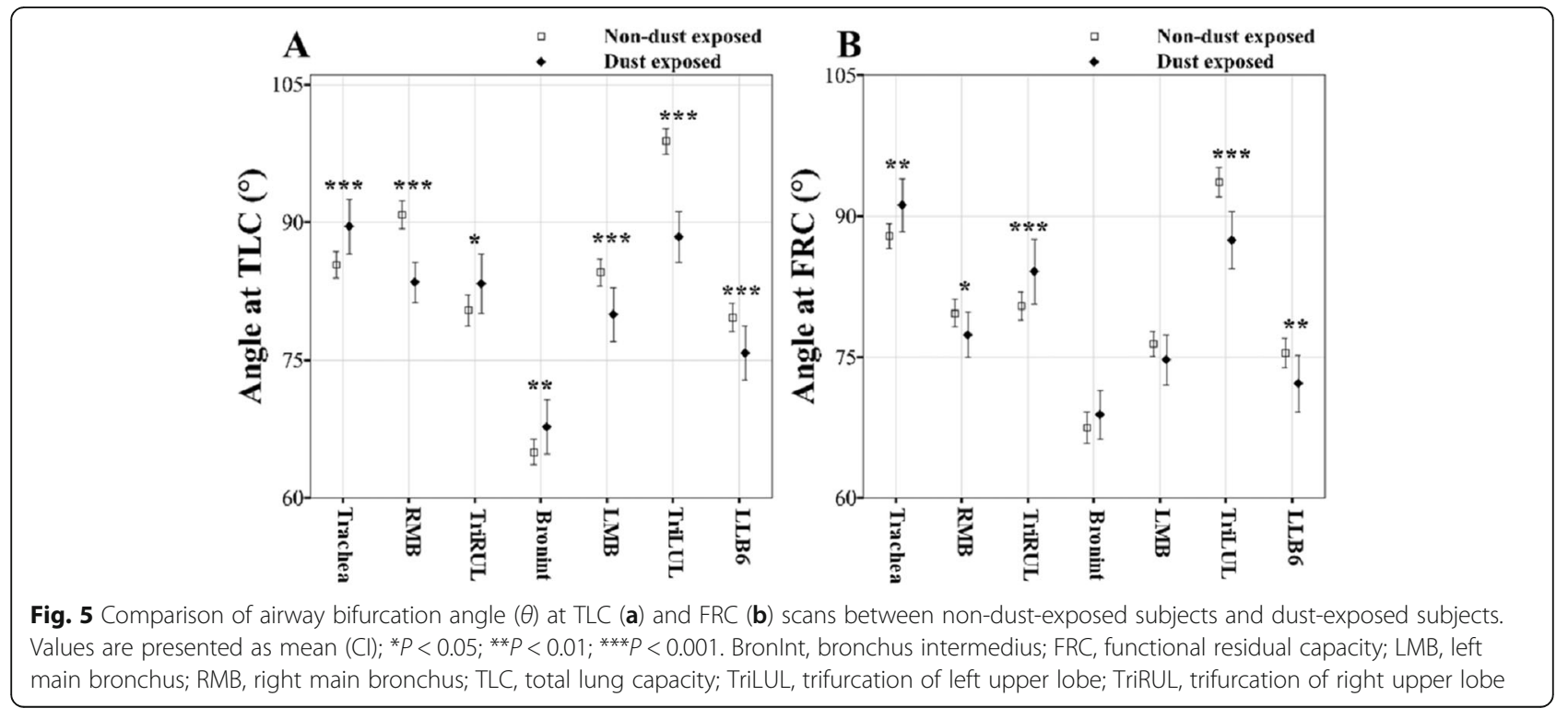



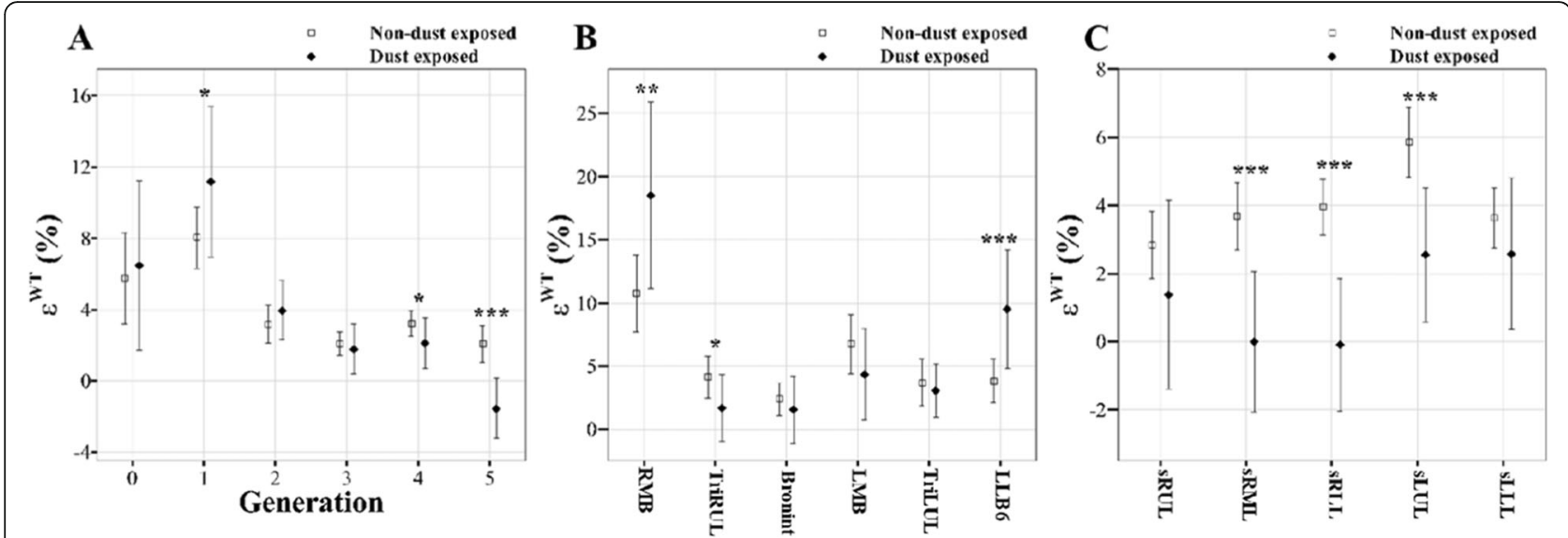

Fig. 6 Comparison of deformation ratio of wall thickness $\left(\varepsilon^{W T}\right)$ between non-dust-exposed subjects and dust-exposed subjects. Values are presented as mean $(\mathrm{Cl}) ;{ }^{*} P<0.05 ;{ }^{* *} P<0.01 ;{ }^{* *} P<0.001$. Generation zero is started from trachea. Bronlnt, bronchus intermedius; FRC, functional residual capacity; LMB, left main bronchus; RMB, right main bronchus; sLLL, subgrouped left lower lobe; sLUL, subgrouped left upper lobe; sRLL, subgrouped right lower lobe; SRML, subgrouped right middle lobe; SRUL, subgrouped right upper lobe; TLC, total lung capacity; TriLUL,

trifurcation of left upper lobe; TriRUL, trifurcation of right upper lobe

and $\mathrm{SSAD} \%$ [22], whereas they are less sensitive on airway size parameters [20]. Therefore, we employed a fraction threshold method to compute the Emph\% and fSAD\%.

With sensitive QCT imaging metrics, we demonstrated that the airway structures of DE subjects had different features from those of NDE subjects. The DE subjects are characterized by phenotypes of airway narrowing $\left(D_{\mathrm{h}}\right)$ at lower-lobes, wall thickening (WT) at all segmental airways, and alteration of branching structure $(\theta)$ at central airways. These findings were similarly observed in a previous study where individuals with occupational exposure had an increased airway wall thickness [9]. In the meantime, a multicenter study of former and current smokers using the multi-ethnic study of atherosclerosis (MESA) COPD data reveals that COPD subjects caused by mainly smoking have thinner airway

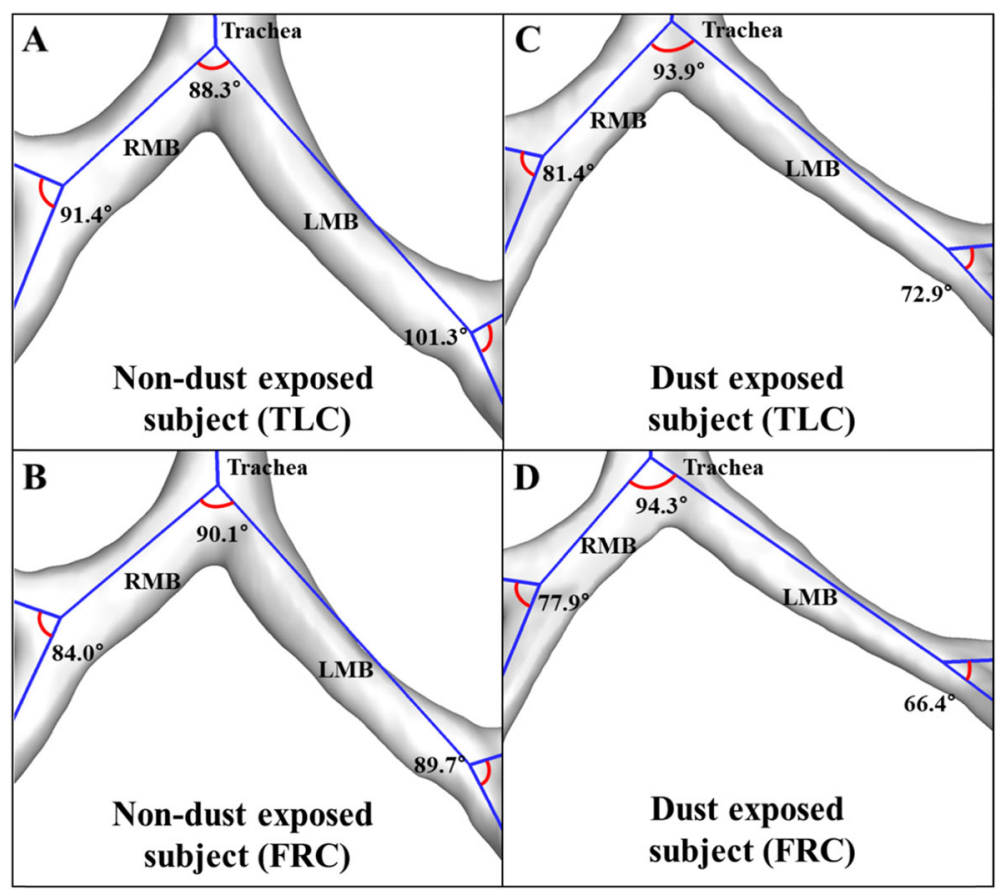

Fig. 7 Bifurcation angle of airway in a non-dust-exposed subject at TLC (a) and FRC (b) scans and a dust-exposed subject at TLC (c) and FRC (d) scans. FRC, functional residual capacity; LMB, left main bronchus; RMB, right main bronchus; TLC, total lung capacity 


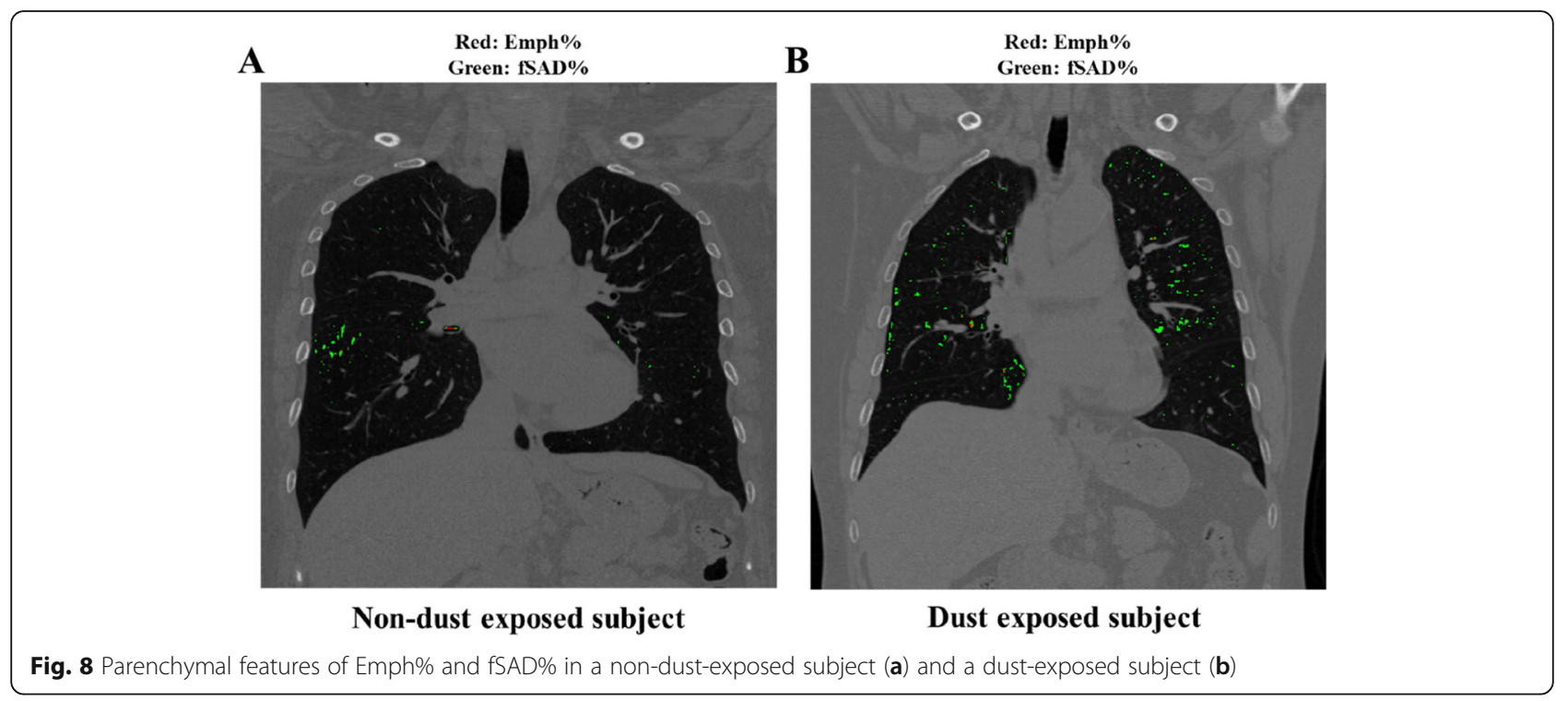

walls [23]. The subjects in this study could be also progressed into COPD later, but these subjects exposed by cement dusts have thickened airway walls. A previous study has reported that exposure to cement dust leads to an increase in airway inflammation [24]. Thus, the distinguished phenotypes on airway walls are likely to indicate different airway pathophysiology.

A recent asthma study by Shim et al. [25] using severe asthma research program (SARP) data has demonstrated an association of airway lumen change between TLC and FRC with a corticosteroid treatment, but there were no investigations of wall thickness and branching angle changes between TLC and FRC. In this study, we computed strains for airway hydraulic diameter, wall thickness, and branching angle. To our best knowledge, this is the first effort of estimating strains at bronchial levels between TLC and FRC. As a result, the DE subjects were found to have the increased stiffness of wall thickness $\left(\varepsilon^{\mathrm{WT}}\right)$ and bifurcation angle $\left(\varepsilon^{\theta}\right)$ which could be affected by lung fibrosis and atelectasis, possibly due to the airway inflammation. In particular, the stiffened airways were likely to affect the prevention of airway deformation from FRC to TLC, sustaining the airway skeletal structure at FRC.

Regarding parenchymal functional variables (Table 4), lung volume at TLC, lung volume at FRC, IC, and Jacobian in DE subjects were smaller than NDE subjects. The decreased IC and Jacobian in DE subjects also could indicate a reduction of lung deformation. Especially, the reduction of Jacobian was found to be significantly correlated with $\varepsilon^{\theta}$ at RMB (Spearman test $R=0.416, P<$ $0.005)$. Based upon our analysis, we presume that the significantly reduced lung volume at TLC was caused by a reduced volume change (Jacobian). This is also possibly correlated with an increased stiffness of airways estimated by $\varepsilon^{\mathrm{WT}}$ and $\varepsilon^{\theta}$ (Table 3 and Fig. 6). In this study,
fSAD\% of DE subjects was similar with NDE subject, and Emph\% of DE subjects was even lower than NDE subjects (Table 4). This is possibly due to the subgrouping by normal lung function, and also indicates that structural alterations of segmental airways begin earlier than parenchymal functional alterations.

Compared with lung functional metrics, airway structural variables provided very clear differences between the DE and NDE subjects. This implies that dust exposure due to cements was significantly associated with bronchial alterations in segmental scales rather than in parenchymal levels. As the size of cement dust ranges from 0.5 to $5 \mu \mathrm{m}$ [16], cement particles may be deposited in segmental airways $[24,26,27]$. These features are different from the characteristics of cigarette smoke particles. Sahu et al. [28] demonstrated that the deposition rate of cigarette smoke particles was greater in parenchymal regions than in segmental regions due to the small size of the particles (ranging from 0.01 to $1 \mu \mathrm{m}$ ). A previous study found that smokers with normal spirometry were more susceptible to parenchymal alteration associated with the emphysema score $[29,30]$. Whether the structural alterations observed here progress to parenchymal levels, leading to severe airtrapping and emphysema, has yet to be confirmed with a longitudinal study.

This study has several limitations. It was retrospectively designed by utilizing CT images collected at two respective sites. Thus, the findings obtained here were possibly influenced by intersite variability, such as scanner difference. However, as shown in Table 1, the two centers used the same scanner make (Siemens), same inspiratory maneuver (TLC), same expiratory maneuver (FRC), and similar reconstruction algorithms (B30f from $\mathrm{KNUH}$ and B35f from $\mathrm{CNUH}$ ), so consistent regional attenuation, airway diameter, and wall thickness between 
the two groups are expected [31, 32]. In addition, the percent emphysema and percent $\mathrm{FSAD}$ were derived from the method using a fraction-threshold [10] that is a density variation-free method. Therefore, these results were not significantly influenced by scanner differences. In the previous study [20,33], we already confirmed that different scanner had little confounding effect for QCT analysis with data derived from different sites. Furthermore, dust-exposed subjects could suffer from several pulmonary diseases such as interstitial lung disease and fibrosis which were not indicated by $\mathrm{FEV}_{1}$ and FVC. Therefore, it was better to include DLCO for the criterion when choosing subjects with normal lung function. Unfortunately, DLCO was not collected in this project, but we excluded any noticeable parenchymal diseases such as fibrosis, asthma, and pneumonia, so we believe that the current features in cement dust exposed subjects remained the same.

\section{Conclusions}

In conclusion, with QCT imaging metrics, we demonstrated that DE subjects had unique features of airway structure, especially in segmental airways, compared with NDE subjects. In structural variables, DE subjects showed airway narrowing at lower-lobes, wall thickening at all segmental airways, a different bifurcation angle at central airways, and a loss of airway wall elasticity at lower-lobes compared with NDE subjects. Unlike segmental airways, parenchymal changes were relatively marginal at this stage for subjects with normal spirometry, which may be associated with the large size of cement dust. It has yet to be investigated if airway structural changes are associated with flow structure and particle distribution and deposition, so a future study with computational fluid dynamics is needed.

\footnotetext{
Abbreviations

QCT: Quantitative computed tomography; $D_{\mathrm{h}}$ : Hydraulic diameter; WT: Wall thickness; $\theta$ : Bifurcation angle; TLC: Total lung capacity; FRC: Functional residual capacity; COPD: Chronic obstructive pulmonary disease; FEV $_{1}$ : Forced expiratory volume in one second; Jacobian: Determinant of Jacobian; fSAD\%: Percent functional small airway disease; Emph\%: Percent emphysema; DE: Dust-exposed; NDE: Non-dust-exposed; KNUH: Kangwon national university hospital; CODA: Chronic obstructive pulmonary disease in dusty areas near cement plants; $\mathrm{CNUH}$ : Chonbuk national university hospital; FVC: Forced vital capacity; PSM: Propensity score matching; PFT: Pulmonary function test; BMl: Body mass index; $\varepsilon^{D h}$ : Deformation ratio of $D_{h}$; $\varepsilon^{W T}$ : Deformation ratio of WT; $\varepsilon^{\theta}$ : Deformation ratio of $\theta ; \mathrm{RMB}$ : Right main bronchus; LMB: Left main bronchus; RUL: Right upper lobe; RML: Right middle lobe; RLL: Right lower lobe; LUL: Left upper lobe; LLL: Left lower lobe; Bronlnt: Right intermediate bronchus; TriLLB: Trifurcation of left lower lobe; sRUL: Sub-grouped right upper lobe with branches of RB1 to RB3; SRML: Sub-grouped right middle lobe with branches of RB4 to RB5; sRLL: Sub-grouped right lower lobe with branches of RB6 to RB10; sLUL: Subgrouped left upper lobe with branches of LB1 to LB5; sLLL: Sub-grouped left lower lobe with branches of LB6, and LB8 to LB10; IC: Inspiratory capacity; SD: Standard deviation; Cl: Confidence interval; MESA: Multi-ethnic study of atherosclerosis; SARP: Severe asthma research program
}

\section{Authors' contributions}

Conception and design: T.K., H.B.C., S.C.; acquisition of data: W.J.K., C.H.L., K.J.C., S.H.B., S.O.K., G.Y.J; analysis and interpretation of data: all authors; drafting the article or revising it critically for important intellectual content: T.K., H.B.C.S.C., S.H.C., E.A.H., C.L.L.; final approval of the version to be published: all authors.

\section{Funding}

This study was supported by the Korea Ministry of Environment (MOE) as "The Environmental Health Action Program" [RE201806039, and RE201806027], Basic Science Research Program through the National Research Foundation of Korea funded by the Ministry of Education [NRF2017R1D1A1B03034157]

\section{Availability of data and materials} Not applicable.

\section{Ethics approval and consent to participate}

Both the KNUH and $\mathrm{CNUH}$ studies were approved by the Institutional Review Board at individual sites (KNUH 2019-06-007 and CUH 2016-03-020005).

\section{Consent for publication}

Not applicable.

\section{Competing interests}

Eric A. Hoffman is a shareholder in VIDA diagnostics, a company that is commercializing lung image analysis software derived by the University of lowa lung imaging group. He is also a member of the Siemens $C T$ advisory board.

\section{Author details}

${ }^{1}$ School of Mechanical Engineering, Kyungpook National University, 80 Daehak-ro, Buk-gu, Daegu 41566, South Korea. ${ }^{2}$ Department of Internal Medicine and Environmental Health Center, Kangwon National University Hospital, School of Medicine, Kangwon National University, Chuncheon, South Korea. ${ }^{3}$ Department of Radiology, College of Medicine, Seoul National University, Seoul, South Korea. ${ }^{4}$ Department of Radiology, College of Medicine, The University of lowa, lowa City, lowa, USA. ${ }^{5}$ Department of Radiology, Research Institute of Clinical Medicine of Chonbuk National University-Biomedical Research Institute of Chonbuk National University Hospital, Jeonju, South Korea. ${ }^{6}$ Department of Statistics, Kyungpook National University, Daegu, South Korea. ${ }^{7} I H R$-Hydroscience and Engineering, The University of lowa, lowa City, lowa, USA. ${ }^{8}$ Department of Medical Humanities and Social Medicine, College of Medicine, Kosin University, Busan, South Korea.

Received: 4 March 2020 Accepted: 19 May 2020

Published online: 29 May 2020

\section{References}

1. Fishwick D, Bradshaw LM, D'SOUZA W, Town I, Armstrong R, Pearce N, et al, Chronic bronchitis, shortness of breath, and airway obstruction by occupation in New Zealand. Am J Respir Crit Care Med. 1997;156(5):1440-6.

2. Kanatani KT, Ito I, Al-Delaimy WK, Adachi Y, Mathews WC, Ramsdell JW. Desert dust exposure is associated with increased risk of asthma hospitalization in children. Am J Respir Crit Care Med. 2010;182(12):1475-81.

3. Heo J, Park HS, Hong Y, Park J, Hong S-H, Bang CY, et al. Serum heavy metals and lung function in a chronic obstructive pulmonary disease cohort. Toxicol Environ Heal Sci. 2017;9(1):30-5.

4. Zeleke ZK, Moen BE, Bråtveit M. Cement dust exposure and acute lung function: a cross shift study. BMC Pulmonary Med. 2010;10(1):19.

5. Garshick E, Schenker MB, Dosman JA. Occupationally induced airways obstruction. Med Clinics. 1996;80(4):851-78,

6. Huuskonen O, Kivisaari L, Zitting A, Kaleva S, Vehmas T. Emphysema findings associated with heavy asbestos-exposure in high resolution computed tomography of finnish construction workers. J Occup Health. 2004;46(4):266-71

7. Meijer E, Nij ET, Kraus T, van der Zee J, van Delden O, van Leeuwen M, et al. Pneumoconiosis and emphysema in construction workers: results of HRCT and lung function findings. Occup Environ Med. 2011;68(7):542-6. 
8. Gevenois P-A, Sergent G, De Maertelaer V, Gouat F, Yernault JC, De Vuyst P. Micronodules and emphysema in coal mine dust or silica exposure: relation with lung function. Eur Respir J. 1998;12(5):1020-4.

9. Marchetti N, Garshick E, Kinney GL, McKenzie A, Stinson D, Lutz SM, et al. Association between occupational exposure and lung function, respiratory symptoms, and high-resolution computed tomography imaging in COPDGene. Am J Respir Crit Care Med. 2014;190(7):756-62.

10. Choi S, Hoffman EA, Wenzel SE, Tawhai MH, Yin Y, Castro M, et al. Registrationbased assessment of regional lung function via volumetric $C T$ images of normal subjects vs. severe asthmatics. J Appl Physiol. 2013;1 15(5):730-42.

11. Choi S, Hoffman EA, Wenzel SE, Castro M, Fain S, Jarjour N, et al. Quantitative computed tomographic imaging-based clustering differentiates asthmatic subgroups with distinctive clinical phenotypes. J Allergy Clin Immunol. 2017;140(3):690-700. e8.

12. Choi S, Haghighi B, Choi J, Hoffman EA, Comellas AP, Newell JD, et al. Differentiation of quantitative $C T$ imaging phenotypes in asthma versus COPD. BMJ Open Respir Re. 2017;4(1):e000252.

13. Parsons $L$. Reducing bias in a propensity score matched-pair sample using greedy matching techniques, Proceedings of the twenty-sixth Annual SAS users group international conference 2001. Cary: SAS Institute Inc; 2001. p. 214-26.

14. Kim S, Lim M-N, Hong Y, Han S-S, Lee S-J, Kim WJ. A cluster analysis of chronic obstructive pulmonary disease in dusty areas cohort identified three subgroups. BMC Pulmonary Med. 2017;17(1):209.

15. Han Y, Heo Y, Hong Y, Kwon SO, Kim WJ. Correlation between physical activity and lung function in dusty areas: results from the chronic obstructive pulmonary disease in dusty areas (CODA) cohort. Tuberc Respir Dis. 2019;82(4):311-8.

16. Kalačić I. Chronic nonspecific lung disease in cement workers. Arch Environ Health. 1973;26(2):78-83.

17. Kim SS, Jin GY, Li YZ, Lee JE, Shin HS. CT quantification of lungs and airways in normal Korean subjects. Korean J Radiol. 2017;18(4):739-48.

18. Miller MR, Hankinson J, Brusasco V, Burgos F, Casaburi R, Coates A, et al. Standardisation of spirometry. Eur Respir J. 2005;26(2):319-38.

19. Galbán CJ, Han MK, Boes JL, Chughtai KA, Meyer CR, Johnson TD, et al. Computed tomography-based biomarker provides unique signature for diagnosis of COPD phenotypes and disease progression. Nat Med. 2012; 18(11):1711.

20. Choi S, Hoffman EA, Wenzel SE, Castro M, Fain SB, Jarjour NN, et al. Quantitative assessment of multiscale structural and functional alterations in asthmatic populations. J Appl Physiol. 2015;118(10):1286-98.

21. Zhang Z, Kim HJ, Lonjon G, Zhu Y. Balance diagnostics after propensity score matching. Ann Transl Med. 2019;7(1):16.

22. Choi S, Hoffman EA, Wenzel SE, Castro M, Lin C-L. Improved CT-based estimate of pulmonary gas trapping accounting for scanner and lungvolume variations in a multicenter asthmatic study. J Appl Physiol. 2014; 117(6):593-603.

23. Smith BM, Hoffman EA, Rabinowitz D, Bleecker E, Christenson S, Couper D, et al. Comparison of spatially matched airways reveals thinner airway walls in COPD. The multi-ethnic study of atherosclerosis (MESA) COPD study and the subpopulations and intermediate outcomes in COPD study (SPIROMICS). Thorax. 2014;69(11):987-96.

24. Fell AKM, Notø H, Skogstad M, Nordby K-C, Eduard W, Svendsen MV, et al. A cross-shift study of lung function, exhaled nitric oxide and inflammatory markers in blood in Norwegian cement production workers. Occup Environ Med. 2011;68(11):799-805.

25. Shim SS, Schiebler ML, Evans MD, Jarjour N, Sorkness RL, Denlinger LC, et al. Lumen area change (Delta Lumen) between inspiratory and expiratory multidetector computed tomography as a measure of severe outcomes in asthmatic patients. J Allergy Clin Immunol. 2018;142(6):1773-80. e9.

26. Lambert AR, O'shaughnessy PT, Tawhai MH, Hoffman EA, Lin C-L. Regional deposition of particles in an image-based airway model: large-eddy simulation and left-right lung ventilation asymmetry. Aerosol Sci Technol. 2011;45(1):11-25.

27. Usmani OS, Biddiscombe MF, Barnes PJ. Regional lung deposition and bronchodilator response as a function of $\beta 2$-agonist particle size. Am J Respir Crit Care Med. 2005;172(12):1497-504.

28. Sahu S, Tiwari M, Bhangare R, Pandit G. Particle size distribution of mainstream and exhaled cigarette smoke and predictive deposition in human respiratory tract. Aerosol Air Qual Res. 2013;13(1):324-32.
29. Hoesein FAM, de Hoop B, Zanen P, Gietema $H$, Kruitwagen $C L$, van Ginneken $B$, et al. CT-quantified emphysema in male heavy smokers: association with lung function decline. Thorax. 2011;66(9):782-7.

30. Ashraf H, Lo P, Shaker SB, de Bruijne M, Dirksen A, Tønnesen P, et al. Shortterm effect of changes in smoking behaviour on emphysema quantification by CT. Thorax. 2011;66(1):55-60.

31. Kim N, Seo JB, Song K-S, Chae EJ, Kang S-H. Semi-automatic measurement of the airway dimension by computed tomography using the full-widthhalf-maximum method: a study on the measurement accuracy according to the CT parameters and size of the airway. Korean J Radiol. 2008;9(3):226-35.

32. Sieren JP, Newell JD Jr, Barr RG, Bleecker ER, Burnette N, Carretta EE, et al. SPIROMICS protocol for multicenter quantitative computed tomography to phenotype the lungs. Am J Respir Crit Care Med. 2016;194(7):794-806.

33. Cho HB, Chae KJ, Jin GY, Choi J, Lin C-L, Hoffman EA, et al. Structural and functional features on quantitative chest computed tomography in the Korean Asian versus the white American healthy non-smokers. Korean J Radiol. 2019;20(7):1236-45.

\section{Publisher's Note}

Springer Nature remains neutral with regard to jurisdictional claims in published maps and institutional affiliations.

Ready to submit your research? Choose BMC and benefit from:

- fast, convenient online submission

- thorough peer review by experienced researchers in your field

- rapid publication on acceptance

- support for research data, including large and complex data types

- gold Open Access which fosters wider collaboration and increased citations

- maximum visibility for your research: over $100 \mathrm{M}$ website views per year

At BMC, research is always in progress.

Learn more biomedcentral.com/submissions 